\title{
NASOPHARYNGOSCOPY VERSUS PLAIN RADIOGRAPHY IN ADENOID SIZE ASSESSMENT
}

\author{
Safaa Khalaf Faleh*, Husam Haider Salman ${ }^{\circledR}$ \& Haidar Mohammed \\ Salih Al-Attar \\ ${ }^{*}$ MB,ChB, FICMS, Otolaryngologist, ENT Dept., Al-Fayhaa Teaching Hospital, Basrah. ${ }^{\circledR}$ FRCS, DLO, \\ Consultant otolaryngologist, Basrah.^MB,ChB, FICMS, Otolaryngologist, Al-Basrah Teaching Hospital, \\ Basrah, IRAQ.
}

\begin{abstract}
This is a prospective comparative study of adenoid size assessment by using radiography versus nasopharyngoscopy using examination of the adenoid under general anesthesia (GA) as a standard method. This study was conducted on 35 children presented with signs and symptoms of adenoid hypertrophy. All patients were examined by both lateral radiograph view of the postnasal space and nasopharyngoscopy, then both results were compared.

The results for small size adenoid can not be calculated because of small sample size. For medium sized adenoid, the sensitivity for radiography and nasopharyngoscopy are $36.36 \%$, $54.54 \%$ respectively, the specificity was $47.36 \%, 84.21 \%$ respectively and the accuracy was $43.33 \%, 73.33 \%$ respectively. For large sized adenoid the sensitivity for radiography and nasopharyngoscopy was $38.89 \%, 83.33 \%$ respectively, the specificity was $100 \%, 66.66 \%$ respectively, and the accuracy was $63.33 \%, 76.66 \%$ respectively.

In conclusion, nasopharyngoscopy is a simple, safe, repeatable, readily available at the ENT unit, with no radiation hazards, and with negligible trauma. Over all, nasopharyngoscopy has a higher sensitivity, specificity and accuracy than radiography.

Keywords: Adenoid, Radiography, Nasopharyngoscopy, Size assessment.
\end{abstract}

\section{Introduction}

The adenoid or the nasopharyngeal tonsil is located at the junction of the roof and the posterior wall of the postnasal space. The shape of a full sized adenoid is like a truncated pyramid, its apex directed toward nasal septum and its base toward the junction of the posterior wall and the roof of the post nasal space ${ }^{1}$. As adenoid is a lymphoid tissue, it is subjected to hypertrophy during childhood with subsequent involution in adulthood $^{2}$. Recurrent upper respiratory tract infection may cause adenoiditis plus physiological hypertrophy that may lead to nasal obstruction, running nose, mouth breathing, snoring and obstructing sleep apnea $^{3}$. Various methods are used in assessing the adenoid hypertrophy such as posterior rhinoscopic mirror examination, endoscopic assessment, and radiological investigations ${ }^{4}$. Adenoidectomy is one of the common surgical procedures performed on children. Symptomatic adenoiditis with significant adenoid hypertrophy are the criteria used for the indication of adenoidectomy $^{5}$. In this study, we are attempting to validate both radiological assessment (lateral post nasal space plain X-ray film) and flexible fibreoptic nasopharyngoscopy for adenoid size assessment in comparison with examination under anesthesia of the postnasal space as a base line measurement for the fore mentioned two tools.

\section{Patients and Methods}

Thirty five patients were examined at ENT unit, Basrah General Hospital from August 2007 to April 2008. All of them were suffering from symptoms of adenoid hypertrophy (mouth breathing, snoring, 
nasal obstruction, nasal discharge, sleep disturbances, and hearing problems). On examination search were done for signs of adenoid hypertrophy (adenoid facies, nasal obstruction, nasal discharge, and postnasal drip). All patients were sent for lateral X-ray film of the postnasal space to evaluate the adenoid size and then examined by fiberoptic nasopharyngoscopy. After that all patients underwent adenoid size assessment under general anesthesia. The results of both X-ray films and nasopharyngoscopy were compared with the EUA of the postnasal space as the standard objective measure for adenoid size assessment. One child was excluded due to anatomical obstruction that prevent proper examination (Achondroplasia), another 4 children cannot tolerate examination with parental refusal were excluded from the study, so the net results is 30 children were included in this study.

A plain X-ray lateral view of the postnasal space (Fig.1) was performed for all children, and during radiography the child was asked to inhale in standing position with the mouth closed.

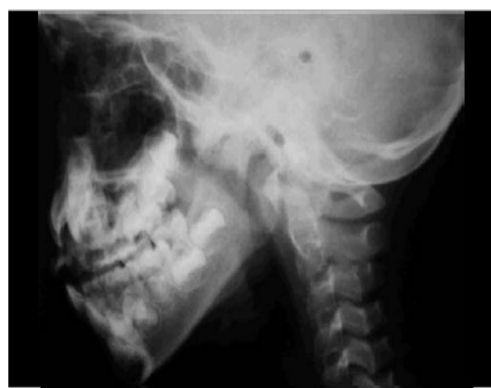

Fig.1: Plain X-ray lateral view of the postnasal space

Interpretation of the X-ray was based on Cohen and Konak (Fig. 2) method in which the soft palate thickness (one centimeter below the hard palate or half centimeter in children younger than three years), and the air column width between the palate and the highest point of convexity of the adenoid were compared ${ }^{6}$.
The adenoid is considered: Small when the column is not narrower than the palates thickness. Medium when the air column is narrower but wider than half of the palates thickness. Large when the air column is narrower than half of the palates thickness.

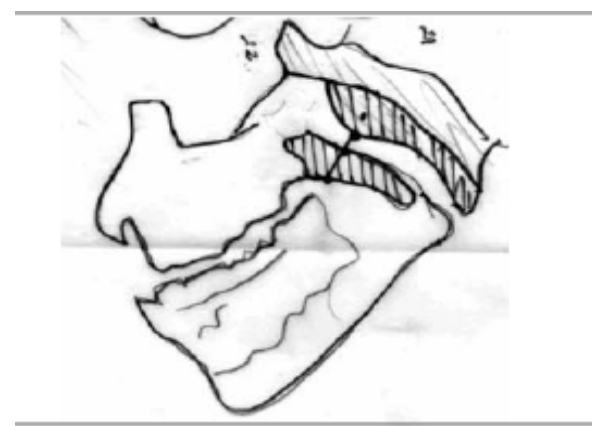

Fig.2: Interpretation of the X-ray was based on Cohen and Konak method.

The flexible nasophapharyngeal examination is started by assessment of the nasal cavity and its structures. The examination was well tolerated by most children especially older ones after reassurance and telling the child that we want to take a picture of his nose. The child is seated in his parent lap with the left arm of the parent bracing the child arms and the right arm placed on the 
forehead, while the legs of the child are held firmly between the legs of his parent, then we apply local anesthesia by spray (2\% Xylocaine) for 5-10 minutes, then the nasopharyngosope was introduced over the floor of the nasal cavity reaching to the choana and adenoid assessed in relation to choanal height (Fig. 3).

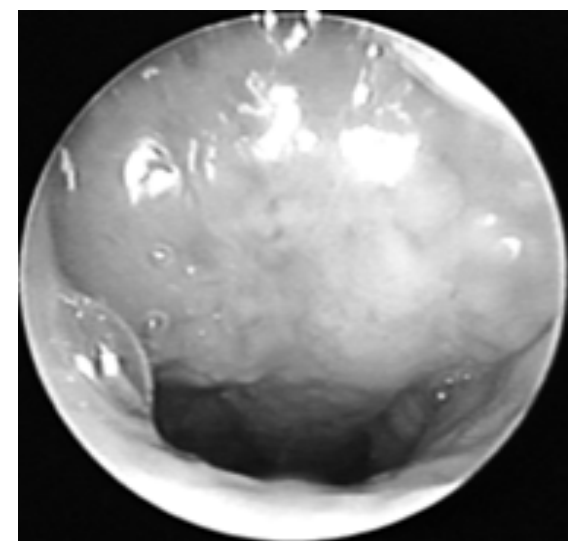

Fig.3: Assessment of the nasal cavity and its structures

Small adenoid; when it occupies less than half of the choana. Medium adenoid; around 50 to $70 \%$ of the choana. Large adenoid; when occupying over $75 \%$ of the choanal area. Assessment of the patient under G.A was done by using a mirror to assess the size of the adenoid in relation to the nasopharynx and the percentage of choanal opening obstruction, and then a finger is slipped behind the soft palate to assess the width of the nasopharynx and adenoid size and choanal opening evaluation ${ }^{7}$. Small: occupy less than half the choana. Medium; $50-70 \%$ of the choana. Large adenoid; when occupy more than $75 \%$ of the choana.

\section{Results}

In EUA of the postnasal space to assess the adenoid size (which is the standard examination in this study) there was only
1 small adenoid (1/30), 11 medium size adenoid (11/30), and 18 large adenoid $(18 / 30)$ (Table I).

Lateral X-ray of the postnasal space to assess the adenoid size showed 9 small adenoid (9/30), 14 medium size adenoid $(14 / 30)$, and 7 large adenoid (7/30), and in fiberoptic nasopharyngoscopy there were 2 small adenoid (2/30), 9 medium size adenoid (9/30), and 19 large adenoid $(19 / 30)$. The analysis of the correlation between the results of lateral X-ray and EUA showed only 1 of the 9 small size adenoid and 4 of the 14 medium size adenoid (by X-ray) correlated with the EUA results, while all the 7 of the 7 large size adenoid (by X-ray) correlated with the EUA results (Fig.4). In the fiberoptic nasopharyngoscopy only one small size adenoid correlated $(1 / 2)$, and 6 medium size adenoid correlated with the EUA (6/9), 15 large size adenoid correlated with the $\operatorname{EUA}(15 / 19)$ (Fig.5). 
Table I: Individual scoring of adenoid size by X-ray, Nasoendoscope and EUA

\begin{tabular}{|r|r|r|r|}
\hline Case No. & X-ray & F. nasoendoscope & EUA under G.A \\
\hline 1 & Medium & Large & Medium \\
\hline 2 & Medium & Large & Large \\
\hline 3 & Large & Large & Large \\
\hline 4 & Medium & Large & Large \\
\hline 5 & Medium & Medium & Medium \\
\hline $\mathbf{6}$ & Medium & Large & Large \\
\hline 7 & Small & Medium & Medium \\
\hline $\mathbf{8}$ & Small & Large & Medium \\
\hline $\mathbf{9}$ & Medium & Large & Large \\
\hline 10 & Medium & Medium & Large \\
\hline 11 & Large & Large & Large \\
\hline 12 & Large & Large & Large \\
\hline 13 & Medium & Large & Large \\
\hline 14 & Large & Large & Large \\
\hline 15 & Small & Small & Small \\
\hline 16 & Small & Large & Medium \\
\hline 17 & Medium & Medium & Medium \\
\hline 18 & Medium & Large & Large \\
\hline 19 & Large & Large & Large \\
\hline 20 & Small & Medium & Medium \\
\hline 21 & Medium & Medium & Large \\
\hline 22 & Large & Large & Large \\
\hline 23 & Small & Medium & Medium \\
\hline 24 & Medium & Large & Large \\
\hline 25 & Small & Medium & Medium \\
\hline 26 & Small & Medium & Large \\
\hline 27 & Medium & Large & Large \\
\hline 28 & Large & Large & Large \\
\hline 29 & Medium & Large & Medium \\
\hline 30 & Small & Small & Medium \\
\hline & & & \\
\hline
\end{tabular}

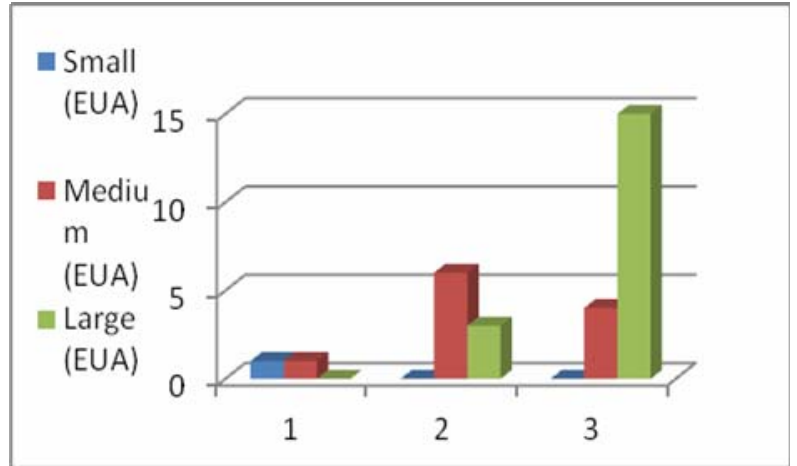

Fig.4: Adenoid size by x-ray in comparison with EUA

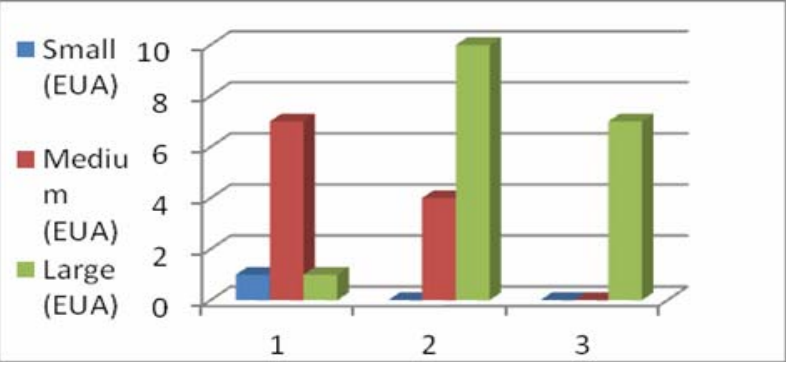

Fig.5: Adenoid size By Endoscope in comparison with EUA 
The average size of adenoid by X-ray (in a 1-3 scale)(Table II) was 1.93, while through nasopharyngoscopy it was 2.57, and during EUA under G.A the average was 2.57. Therefore adenoids evaluated by nasopharyngoscopy were on average $32 \%$ larger as compared to adenoid evaluated by lateral X- Ray. The sensitivity, specificity, and accuracy of the lateral X-ray in relation to EUA under G.A, $n=1$ (small) so cannot be calculated for small size adenoid, for medium size adenoid sensitivity is $36.36 \%$, specificity is $47.36 \%$, and accuracy is $43.33 \%$, and for large adenoid sensitivity is $38.89 \%$, specificity is $100 \%$, and accuracy is $63.33 \%$ (Fig. $6 \& 7$ ).

The sensitivity, specificity, and accuracy of the nasopharyngoscopy in relation to EUA under G.A, because $\mathrm{n}=1$ (small) so cannot be calculated for small size adenoid, for medium size adenoid sensitivity is $54.54 \%$, specificity is $84.21 \%$, and accuracy is $73.33 \%$, and for large adenoid sensitivity is $83.33 \%$, specificity is $66.66 \%$, and accuracy is $76.66 \%$.

Table II: Scoring of adenoid size (1; small, 2; medium, 3; large).

\begin{tabular}{|c|c|c|c|}
\hline Case No. & X-ray & F. nasoendoscope & EUA under G.A \\
\hline 1 & 2 & 3 & 2 \\
\hline 2 & 2 & 3 & 3 \\
\hline 3 & 3 & 3 & 3 \\
\hline 4 & 2 & 3 & 3 \\
\hline 5 & 2 & 2 & 2 \\
\hline 6 & 2 & 3 & 3 \\
\hline 7 & 1 & 2 & 2 \\
\hline 8 & 1 & 3 & 2 \\
\hline 9 & 2 & 3 & 3 \\
\hline 10 & 2 & 2 & 3 \\
\hline 11 & 3 & 3 & 3 \\
\hline 12 & 3 & 3 & 3 \\
\hline 13 & 2 & 3 & 3 \\
\hline 14 & 3 & 3 & 3 \\
\hline 15 & 1 & 1 & 1 \\
\hline 16 & 1 & 3 & 2 \\
\hline 17 & 2 & 2 & 2 \\
\hline 18 & 2 & 3 & 3 \\
\hline 19 & 3 & 3 & 3 \\
\hline 20 & 1 & 2 & 2 \\
\hline 21 & 2 & 2 & 3 \\
\hline 22 & 3 & 3 & 3 \\
\hline 23 & 1 & 2 & 2 \\
\hline 24 & 2 & 3 & 3 \\
\hline 25 & 1 & 2 & 2 \\
\hline 26 & 1 & 2 & 3 \\
\hline 27 & 2 & 3 & 3 \\
\hline 28 & 3 & 3 & 3 \\
\hline
\end{tabular}




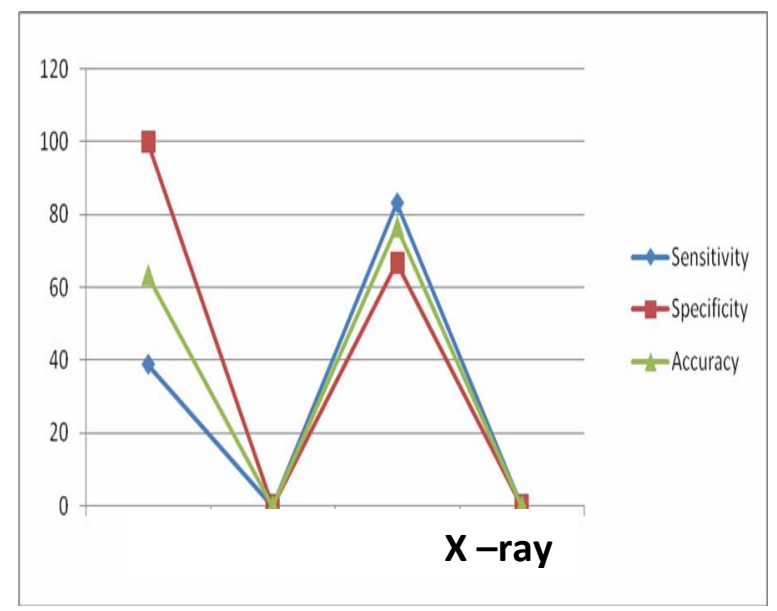

Fig.6: Sensitivity, Specificity, and Accuracy of X-ray and fiberoptic endoscope of large size in relation to EUA.

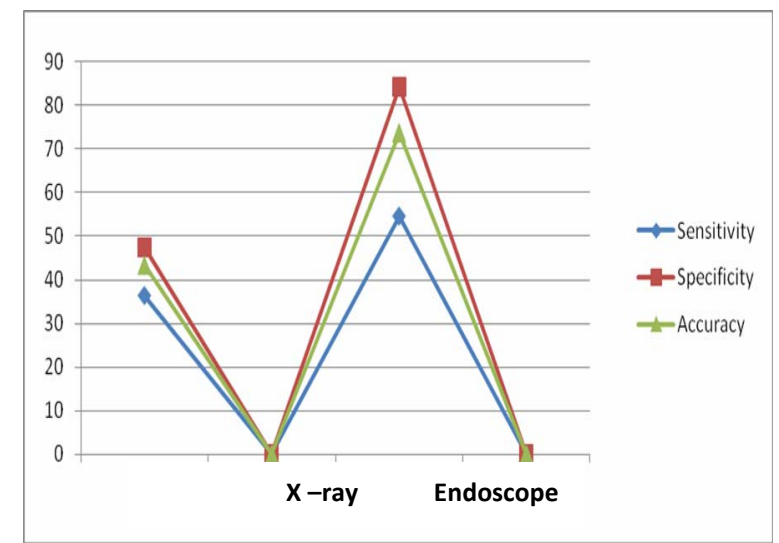

Fig.7: Sensitivity, Specificity, and Accuracy of X-ray and fiberoptic endoscope of medium size in relation to EUA

\section{Discussion}

Clinical evaluation of adenoid size is very difficult ${ }^{8}$. Objective measures of adenoid hypertrophy are useful to provide information that may help in deciding the need of surgery and subsequent outcome evaluation. Cohen and Conak $^{9}$ developed the best approach that takes into account the relation between nasopharynx and adenoid size (need only simple measurements and calculation) and they emphasize that the otolaryngologist should consider the air column rather than the adenoid size or shape, but it has disadvantages that involve radiation exposure to the child with some reports about harmful effect, lack of standardization in technique and film evaluation, beside it is two dimensional image of the nasopharynx and also child refusal and movement during film taking ${ }^{9}$.

Hirschmann firstly performed a nasosinusal endoscopy in 1901. But now great improvement in optical and more comfortable instruments both to the patient and the examiner were developed. The disadvantages of nasopharyngoscopy are during passage of the endoscope injury to the nose, Eustachian tube and adenoid (only 2 cases with simple nasal injury managed without packing), also no reports about standardization of nasopharyngoscopic evaluation (in our study we assessed the size of adenoid in relation to choanal obstruction), another difficulty is non collaborative young children and parent refusal, also endoscopy need good experience. The 
advantages of nasopharyngoscopy are no radiation exposure, easily reproducible, sitting or supine awake or sleeping, widely available and inexpensive ${ }^{10}$.

From the above the correlation between adenoid size by X-ray with EUA results, in small adenoid there is very low correlation while in medium size adenoid there is increase in the correlation, and in large adenoid there is complete correlation between X-ray and EUA results. The correlation between nasopharyngoscopy and EUA. In small adenoid there is lout of 2 is correlated and there is good correlation in medium size adenoid and better correlation in large adenoid but not complete.

The average size of the adenoid by X-ray is 1.93 (in 1-3 scale) and through nasopharyngoscopy the average size 2.57 which is the same in EUA under G.A, so the size of adenoid by fibroendoscopy is on average $32 \%$ larger as compared to $\mathrm{X}$ ray. So the adenoid seen by X-ray is mostly smaller than the operative finding. For medium size adenoid in X-ray and fibroendoscopy sensitivity $36.36 \%$, $54.54 \%$ respectively, specificity is $47.36 \%, \quad 84.21 \%$ respectively, and accuracy is $43.33 \%, 73.33 \%$ respectively. So there is clear advantage of fibroendoscopy over X-ray in all parameters.

For large size adenoid X-ray and fibroendoscopy the sensitivity $38.89 \%$, $83.33 \%$ respectively, specificity $100 \%$, $66.66 \%$ respectively, and accuracy $63.33 \%, 76.66 \%$ respectively. Also there is clear difference in parameters between X-ray and fibroendoscopy with single relevant finding of $100 \%$ specificity for $\mathrm{X}$-ray which mean that when X-ray show large adenoid then it is $100 \%$ large.

Kurien et al evaluated the correlation between X-ray and endoscopy; she considered endoscopy as a gold standard test. She found that 17 out of 26 children has complete correlation between X-ray and endoscopy (65\%) which is higher correlation than our study (43\%). This may be due to the difference in technique of evaluation ${ }^{11}$.

Edmir et al. found that only 8 of 20 children have correlation between X-ray and fiberoptic endoscopy finding $(40 \%)^{12}$, and he found that the sensitivity of X-ray in relation to EUA finding for medium size and large adenoid 20\%, $46.66 \%$ respectively, specificity $66.66 \%$, $100 \%$, and accuracy 55\%, 72\% respectively. There is difference in the results between Edmir et al and our study which may be technical, but also there is $100 \%$ specificity in large size adenoid. $\mathrm{He}$ also found that the sensitivity of fibroendoscopy in relation to EUA in medium and large size adenoid $60 \%, 92 \%$ respectively, specificity $89 \%, 86.5 \%$ respectively, and accuracy $83 \%, 91 \%$ respectively.

There is a clear difference in the parameters in Edmir et al in comparison with our study which may be attributed to difference in experience and the technique used in evaluation ${ }^{12}$.(Fig. 8\&9).

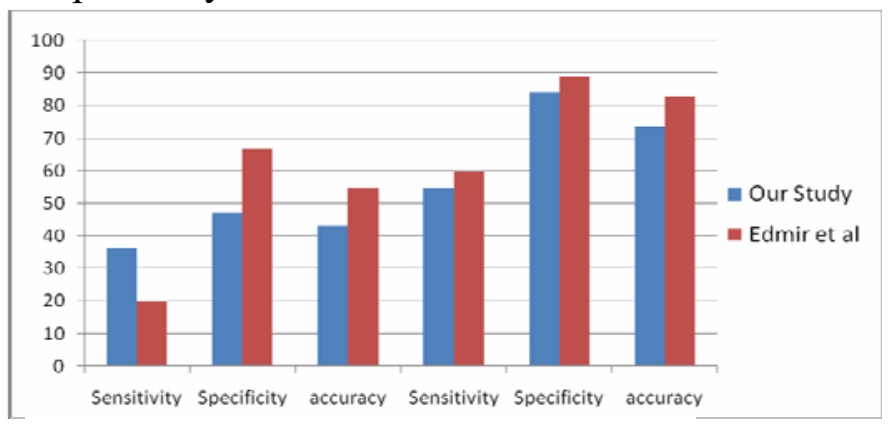

$\mathrm{X}$-ray

Fig.8: Comparison between our results and those for Edmir et al For medium size adenoid 


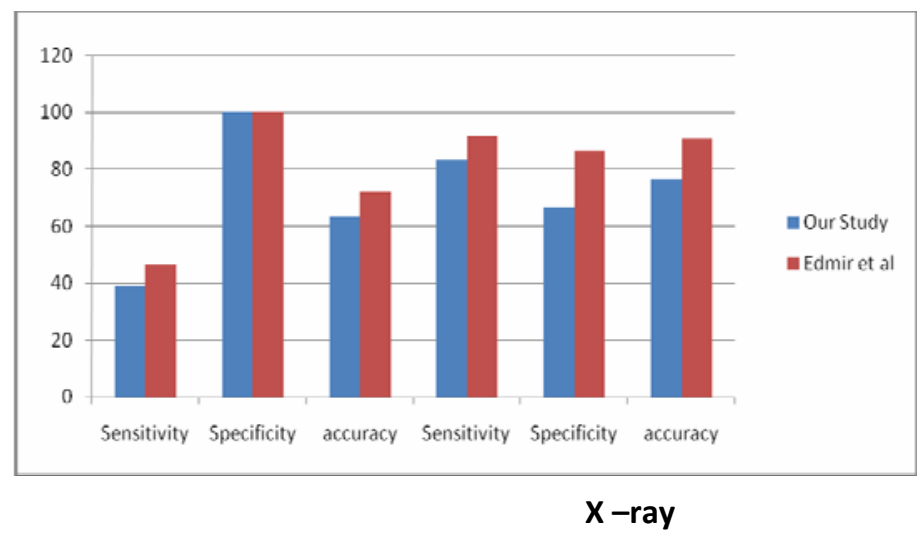

Fig.9: Comparison between our results and those for Edmir et al For large size adenoid

Conclusion: It is clear from our study and other studies that there is a greater advantage of fibroptic nasopharyngoscopy over plain radiography for adenoid size assessment in all the values of significance apart from higher specificity for radiography in diagnosing large adenoid. Also we conclude that clinical evaluation of adenoid by history and physical examination should not be ignored even when there is small adenoid by radiography (only one small adenoid during operative finding). So fibroptic nasopharyngoscopy is a simple, safe, repeatable and readily available at otolaryngology unit with no radiation hazards and extra cost, with negligible trauma to the child and over all higher sensitivity, specificity and accuracy over plain radiography of the adenoid and give comparable results to examination under anaesthesia of adenoid size .

Recommendations: From the results of our study we recommend that every child with a history and physical signs of adenoid hypertrophy should be subjected to nasopharyngoscopy under local aneasthesia to evaluate the adenoid size, and if the endoscope is not available and the radiography was negative and the child still has typical findings in history and physical examination, then he should be assessed under GA to exclude adenoid enlargement.

\section{References}

1. Hamza SB, Ranjith V. T. Assessment of size of adenoid-comparison of adenoidal nasopharyngeal ratio and nasal endoscopy in children with chronic adenoiditis. International Journal of Research in Medical Sciences; 2019 Mar; Vol. 7(3):776- 781

2. Brandtzaeg, P. Immune functions of nasopharyngeal lymphoid tissue. Adv Otorhinolaryngol. 72, 20-24 (2011).

3. Takayoshi Ishida et al. Patterns of adenoid and tonsil growth in Japanese children and adolescents: A longitudinal study; Scientific reports;(2018) 8:17088; DOI:10.1038/s41598-018-35272-z

4. Sharifkashani S et al. A New Clinical Scoring System for Adenoid Hypertrophy in Children; Iranian Journal of Otorhinolaryngology, Vol. 27(1), Serial No.78, Jan 2015:56-61

5. Feres MFN et al. Lateral X-ray view of the skull for the diagnosis of adenoid hypertrophy: A systematic review; International Journal of Pediatric Otorhinolaryngology 75 ;Jan 2011: 1-11

6. Shields G. The tonsil and adenoid in paediatric patients.June 19.2002.UTMB,pp 4.

7. Goldstein N. A. Clinical assessment of paediatric obstructive sleep apnea. Paeditrics vol 114 .1 July, 2004, pp 33-43.

8. Paradise J. L. Assessment of adenoidal obstruction in children,clinical signs versus Roentginogrphic finding. Paeditrics vol ,101. No 6 June 1998. Pp 979-986.

9. Sakano E. Nasal fiberoptic examination for the assessment of adenoid hypertrophy,importance and precautions indiagnosis . Journal De Pediatria. 2005.

10. Chisholm EJ, Lew-Gor S, Hajioff D, Caulfield H. Adenoid size assessment a comparizon of palpation, nasendoscopy and mirror examination. Clinical otolaryngology. 2005.feb ,30 . pp39-41.

11. Semelka R. C. Imaging x ray cause cancer . Medscape. Feb $13,2006$.

12. Lorenzo E. A. Comparison between radiological and nasopharyngoscopic assessment of adenoid tissue volume in mouth breathing children. Rev Bras otolaryngology vol 81 pp 32-44 mar 2006. 